Supplementary Information for:

\title{
Evolution of a protein interaction domain family by tuning conformational flexibility
}

\author{
Dustin S. Whitney ${ }^{\dagger}$, Brian F. Volkman* ${ }^{\dagger}$, Kenneth E. Prehoda** \\ ${ }^{\dagger}$ Department of Biochemistry, Medical College of Wisconsin, Milwaukee, WI 53226 and ${ }^{\star}$ Department of Chemistry \\ and Biochemistry, Institute of Molecular Biology, University of Oregon, OR 97403
}



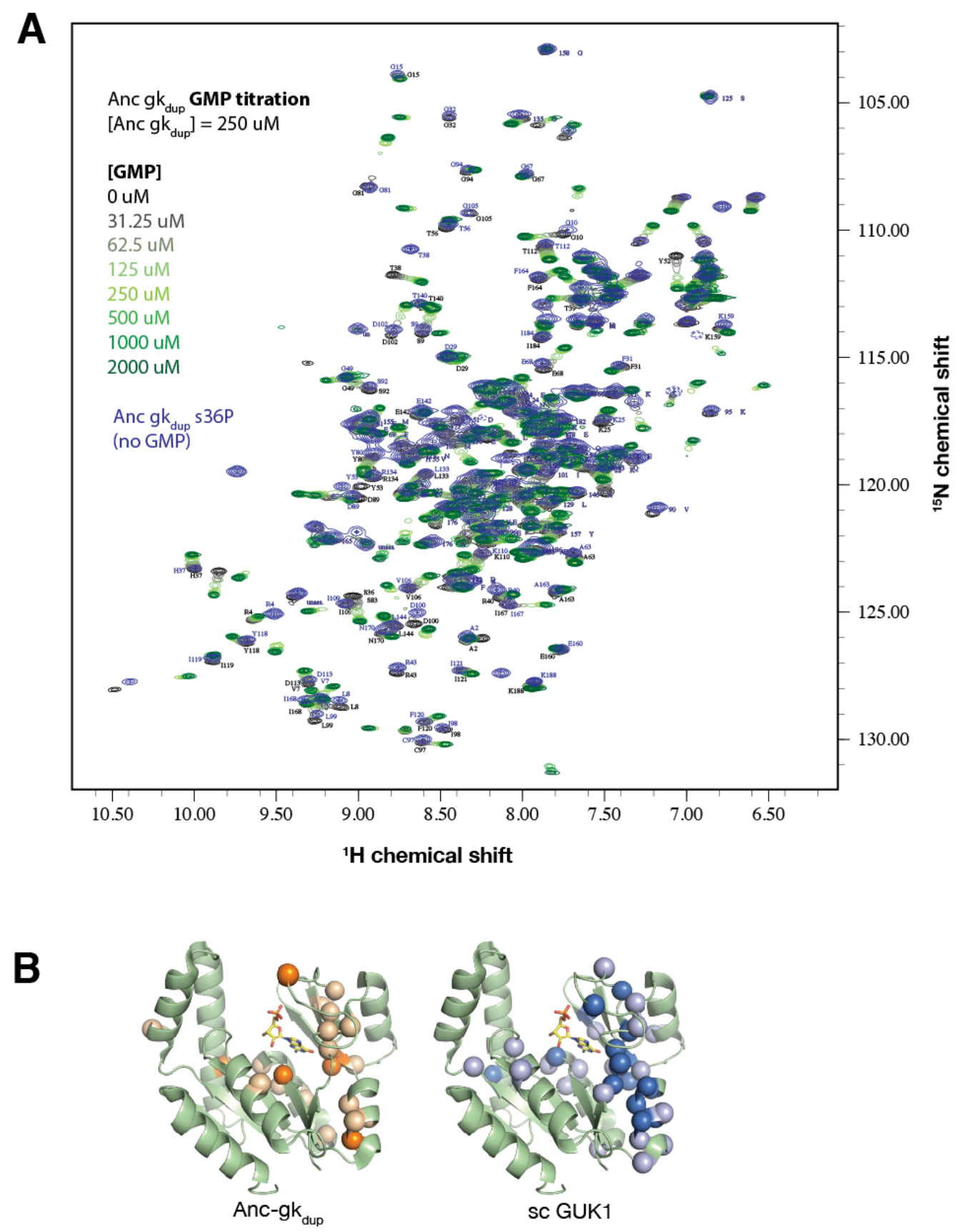

Supplementary Figure 1. Full ${ }^{15} \mathrm{~N}-{ }^{1} \mathrm{H}$ HSQC spectra of Anc-gk $\mathrm{dup}_{\text {dup }}$ GMP titration and Anc-gk $\mathrm{dup}_{\mathrm{s}} \mathbf{3 6 P}$ A. Full ${ }^{1} \mathrm{H}^{-15} \mathrm{~N}$ HSQC NMR spectra of Anc-gk ${ }_{\text {dup }}$ from 0 to $2000 \mu \mathrm{M}$ GMP and Anc-gk dup $\mathrm{s} 36 \mathrm{P}$.

B. Comparison of residues affected by GMP binding in Anc-gk $\mathrm{dup}_{\text {dup }}$ and an extant GK enzyme (yeast GUK1). Residues shifting more than $0.6 \mathrm{ppm}$ upon GMP saturation are colored orange and blue for $\mathrm{Anc}-\mathrm{gk}_{\mathrm{dup}}$ and yeast GUK1, respectively. Residues shifting 0.3-0.59 ppm are colored light orange and light blue for Anc-gk dup and yeast GUK1, respectively. The overlap of these residue positions, even though the homology between these proteins is low, suggests that similar GMP-induced conformational changes occur. The changes are shown on the structure of yeast GUK1 complexed with GMP (PDB 1EX7). 
A

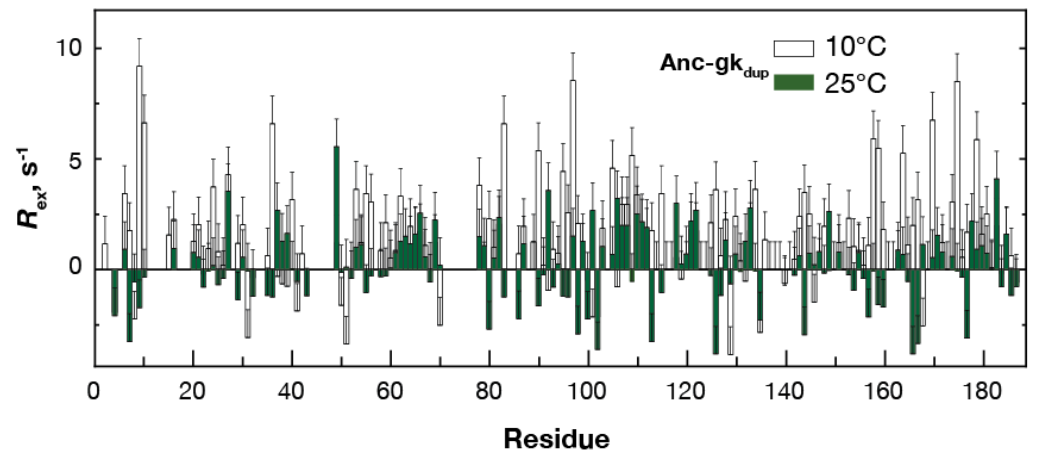

B

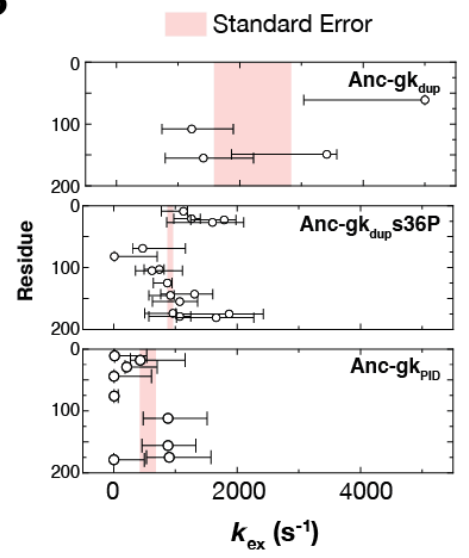

\section{Asp 89}
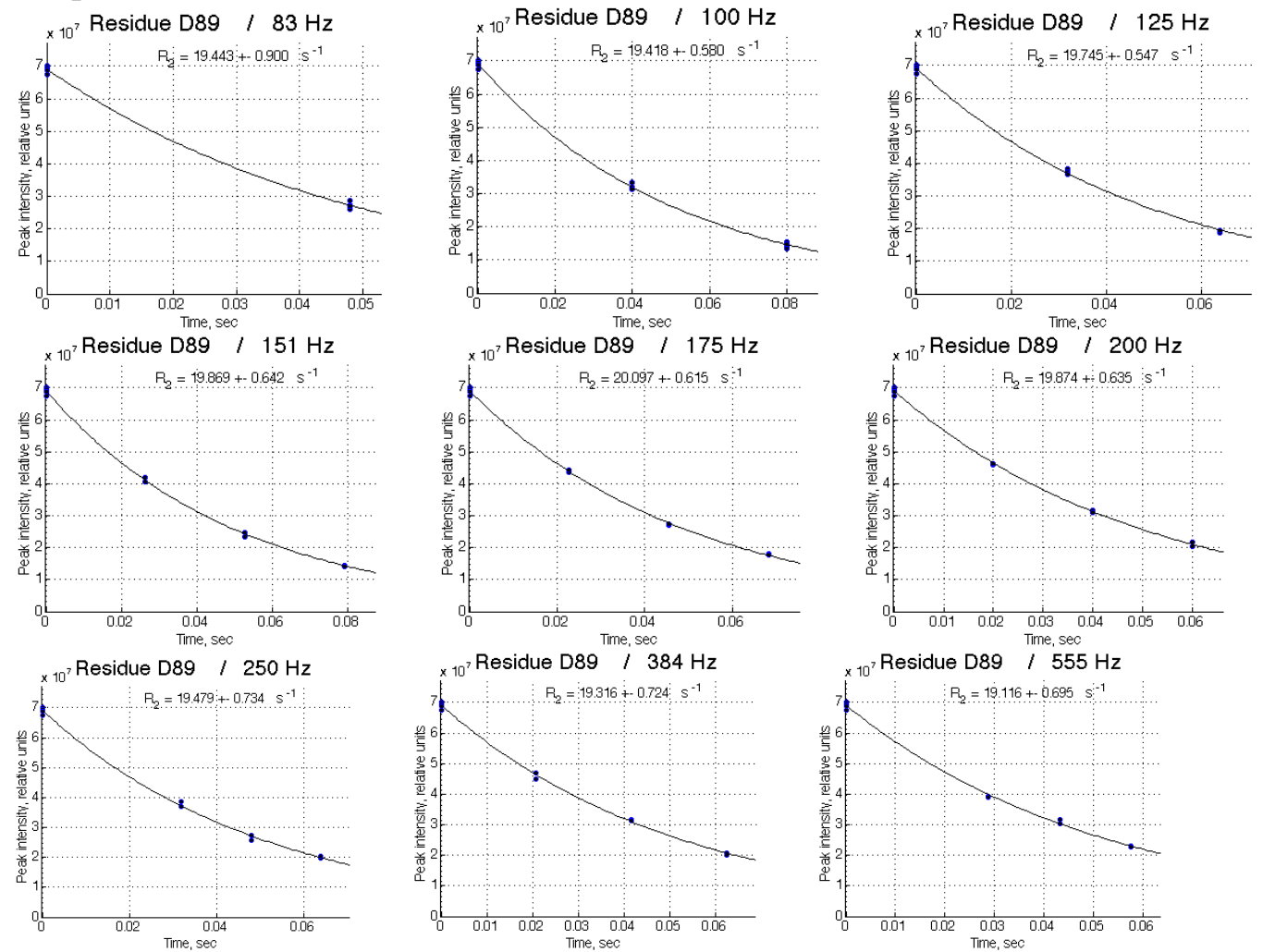

D89, Offset 3.29 ppm, $50.7 \mathrm{MHz}$

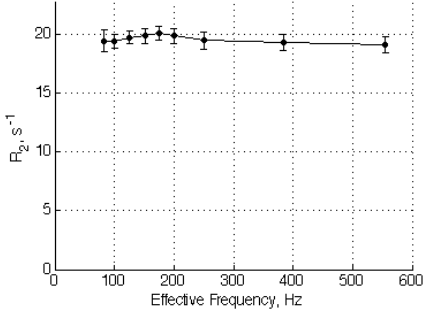




\section{Glu 174}
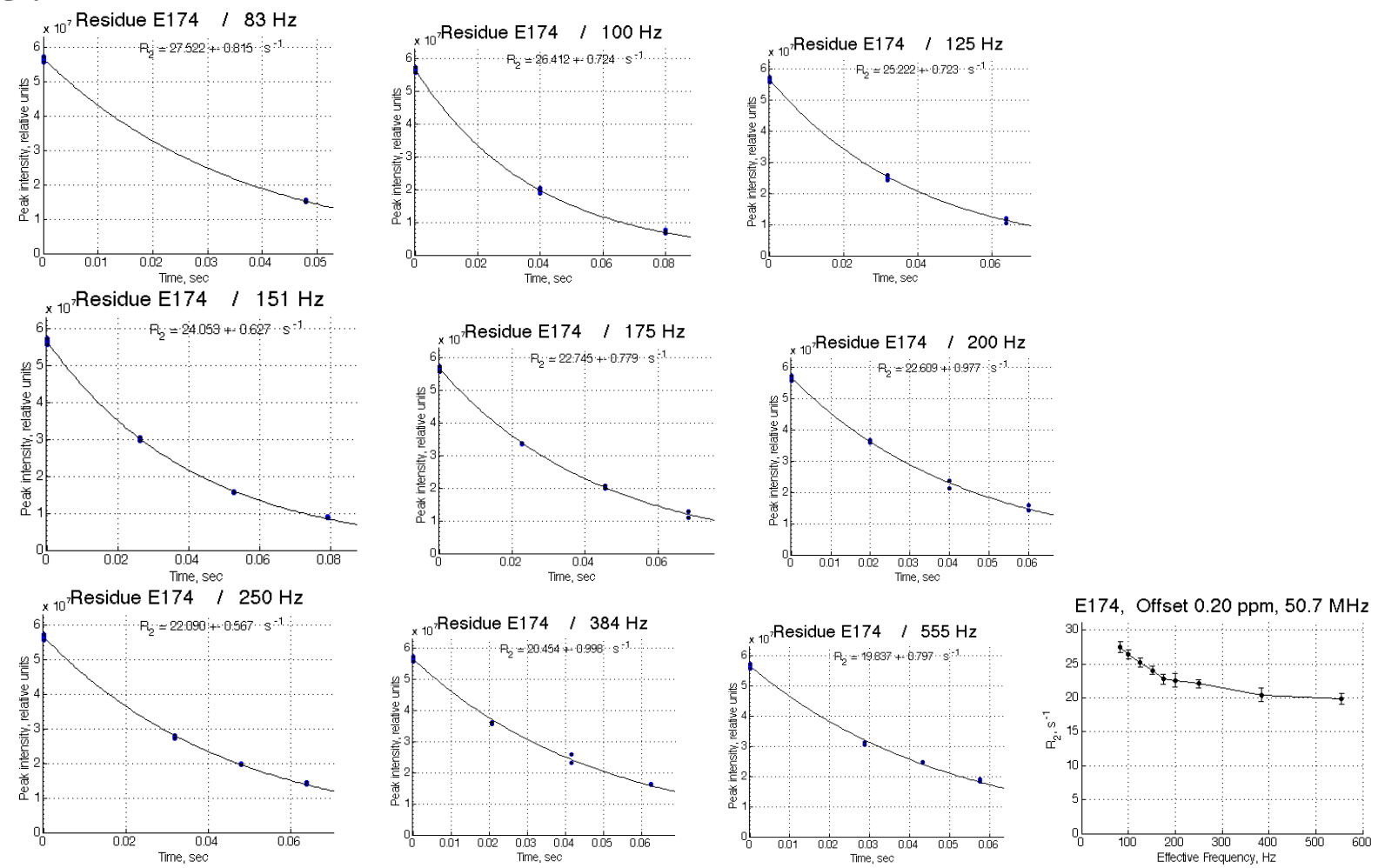

Supplementary Figure 2. Relaxation dispersion data for ancestral GK proteins.

A. $\mathrm{R}_{\mathrm{ex}}$ comparison for Anc-gk $\mathrm{dup}_{\text {at }} 25^{\circ} \mathrm{C}$ and $10^{\circ} \mathrm{C}$. Error bars represent standard error.

B. Change in dynamic behavior from ancestral enzyme to protein interaction domain. The exchange rate constant for the interconversion between open and closed GK, as determined for individual residues, is shown for Anc-gk dup, Anc-gk $\mathrm{k}_{\text {dup }} \mathrm{s} 36 \mathrm{P}$, and Anc-GK $1_{\mathrm{PID}}$ from measurements made at $25^{\circ} \mathrm{C}$. Because the dynamic motions in $A n c-\mathrm{gk}_{\text {dup }}$ are very fast, the exchange rate constant is poorly determined (a small number of Anc-gk $\mathrm{dup}_{\text {dup }}$ residues with $k_{\mathrm{ex}}$ above $5000 \mathrm{~s}^{-1}$ are not shown for clarity). The rate constant is significantly smaller in Anc-gk dup $_{\text {s }} 36 \mathrm{P}$ (middle panel) and even further reduced in Anc-GK $1_{\mathrm{PID}}$ (bottom panel). Error bars represent the propagated standard error from the high and low points of $\mathrm{R}_{2, \text { eff }}$ CPMG frequency response data, such as that shown in panel B. Shaded regions represent the $k_{\text {ex }}$ mean surrounded by the standard error.

C. Initial $\mathrm{R}_{2}$ fits for two representative residues at multiple relaxation times and effective CPMG frequencies. Asp 89 possesses a flat, featureless profile and Glu 174 shows large $\mathrm{R}_{\mathrm{ex}}$. The dispersion curves for these two residues arise from Anc-gk $\mathrm{dup}_{\mathrm{s}} \mathrm{s} 6 \mathrm{P}$ at $25^{\circ} \mathrm{C}, 500 \mathrm{MHz}$ dataset. The final plot shows all fitted $\mathrm{R}_{2 \text {,apparent }}$ values plotted by effective $\mathrm{CPMG}$ frequency. 
A
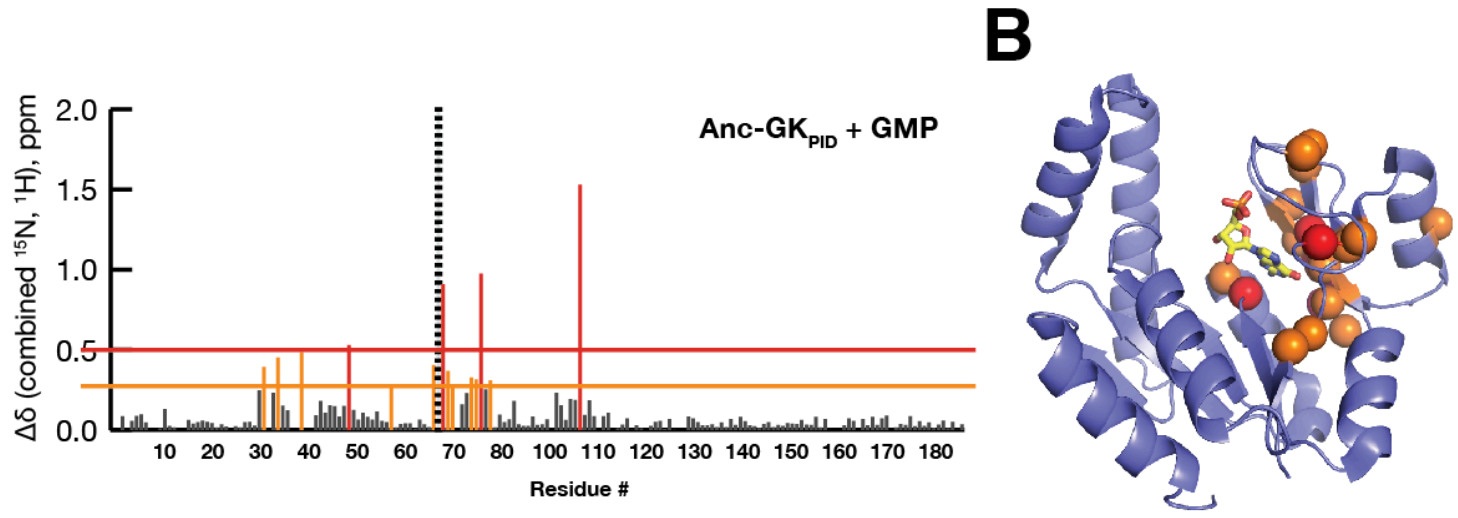

Supplementary Figure 3. Effect of GMP binding on Anc-GK1 $1_{P I D}{ }^{15} \mathrm{~N}^{1} \mathrm{H}$ HSQC NMR signals.

A. Plot of combined ${ }^{15} \mathrm{~N}$ and ${ }^{1} \mathrm{H}$ GMP-induced chemical shift change versus Anc-GK $1_{\text {PID }}$ residue number. Colored lines indicate thresholds for inclusion of residues in (b).

B. Anc-GK1 $1_{\text {IID }}$ residues whose HSQC chemical shifts were perturbed by GMP addition (according to thresholds shown in panel A) are plotted on the GK structure (PDB ID 1EX7). 
A
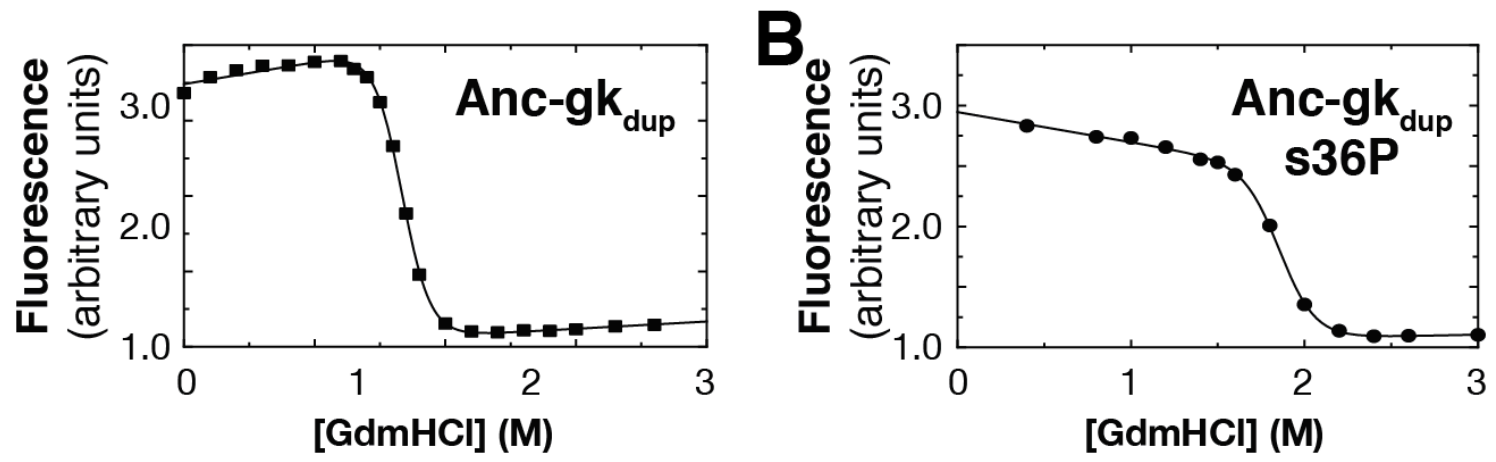

C

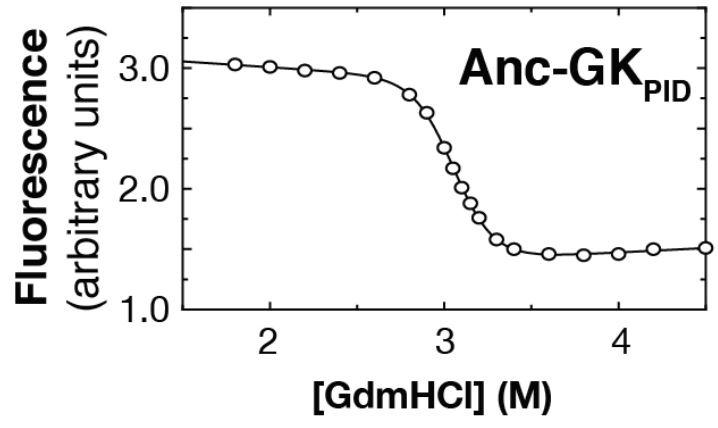

D

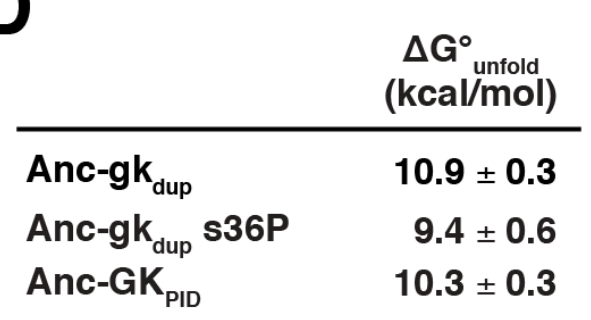

$\Delta G^{\circ}{ }_{\text {unfold }}$

$10.9 \pm 0.3$

Anc-GK

$10.3 \pm 0.3$

Supplementary Figure 4. Stability of ancestral GKs

A-C. Fluorescence of indicated GK proteins as a function of guanidine hydrochloride concentration.

D. Protein stability values $\left(\Delta \mathrm{G}^{\circ}{ }_{\text {unfold }}\right)$ obtained from the fits shown in panels D-F. 